\title{
Progress Toward Polio Eradication — Worldwide, January 2016-March 2018
}

\author{
Farrah Khan ${ }^{1}$; S. Deblina Datta, MD ${ }^{1}$; Arshad Quddus, $\mathrm{MD}^{2}$; John F. Vertefeuille, $\mathrm{PhD}^{1}$; Cara C. Burns, $\mathrm{PhD}^{3}$; Jaume Jorba, PhD ${ }^{3}$; \\ Steven G.F. Wassilak, MD ${ }^{1}$
}

In 1988, when an estimated 350,000 cases of poliomyelitis occurred in 125 countries, the World Health Assembly resolved to eradicate polio globally. Transmission of wild poliovirus (WPV) continues uninterrupted in only three countries (Afghanistan, Nigeria, and Pakistan) (1), and among the three serotypes, WPV type 1 (WPV1) remains the only confirmed circulating type. This report describes global progress toward polio eradication during January 2016-March 2018, and updates previous reports (2). In 2017, 22 WPV1 cases were reported, a $41 \%$ decrease from the 37 WPV1 cases reported in 2016. As of April 24, 2018, eight WPV1 cases have been reported (seven in Afghanistan and one in Pakistan), compared with five cases during the same period in 2017. In Pakistan, continuing WPV1 transmission has been confirmed in multiple areas in 2018 by isolation from wastewater samples. In Nigeria, ongoing endemic WPV1 transmission was confirmed in 2016 (3); although WPV was not detected in 2017 or in 2018 to date, limitations in access for vaccination and surveillance in insurgent-held areas in northeastern Nigeria might permit continued undetected poliovirus transmission. Substantial progress toward polio eradication has continued in recent years; however, interruption of WPV transmission will require overcoming remaining challenges to reaching and vaccinating every missed child. Until poliovirus eradication is achieved, all countries must remain vigilant by maintaining high population immunity and sensitive poliovirus surveillance.

\section{Routine Poliovirus Vaccination Coverage}

Among infants aged 1 year, the estimated global coverage with 3 doses of poliovirus vaccines ( $\mathrm{Pol} 3$, mostly oral poliovirus vaccine $[\mathrm{OPV}]$ ) through routine immunization services was $85 \%$ in 2016 (the most recent year for which data are available). World Health Organization (WHO)/United Nations Children's Fund estimates for Pol3 coverage in 2016 were 73\% in the African Region, 92\% in the Region of the Americas, 80\% in the Eastern Mediterranean Region, 94\% in the European Region, 87\% in the South-East Asia Region, and 95\% in the Western Pacific Region, with heterogeneity in coverage among countries in all regions.* National Pol3 coverage with the third dose of OPV (OPV3) in the three countries with endemic WPV transmission in 2016 was 60\% in Afghanistan, $72 \%$ in Pakistan, and $49 \%$ in Nigeria. OPV3 coverage is

\footnotetext{
*http://apps.who.int/immunization_monitoring/globalsummary/timeseries/ tswucoveragepol3.html.
}

substantially lower in areas of WPV transmission, where children in high-risk mobile populations or areas of conflict are repeatedly missed $(4,5)$. Rarely, in areas with low vaccination coverage, Sabin-like viruses can spread and revert to neurovirulence, resulting in outbreaks of disease caused by circulating vaccine-derived polioviruses (cVDPV). Approximately $90 \%$ of cVPDV cases reported since 2006 have been caused by type 2 (cVDPV2). In countries with recent cVDPV detections, Pol3 coverage was $74 \%$ in the Democratic Republic of the Congo (DRC), $48 \%$ in Syria, $47 \%$ in Somalia, and 83\% in Laos (6). In these countries, OPV3 coverage was substantially lower in subnational areas with cVDPV emergence and transmission.

Following certification of the eradication of WPV type 2 (WPV2) in 2015, a global, synchronized withdrawal of trivalent OPV (tOPV, containing types 1, 2, and 3 live, attenuated polioviruses), and switch to bivalent OPV (bOPV, containing types 1 and 3 only), was completed by the end of April 2016 (7). Starting in 2015, injectable trivalent inactivated poliovirus vaccine (IPV) was introduced into routine immunization schedules in OPV-using countries, generally at 14 weeks of age. Some countries had to delay introduction of IPV until 2018 because of global shortages of the vaccine.

\section{Supplementary Immunization Activities}

In 2016, 186 supplementary immunization activities (SIAs) were conducted in five WHO regions, during which approximately two billion total OPV and IPV doses were administered (Table 1), including 1,264,552,301 (63\%) doses administered during national immunization days, $710,995,110$ (36\%) during subnational immunization days, and 17,603,036 (1\%) doses during focused SIAs in areas of known or suspected poliovirus circulation ("mop-up" activities). In the event of cVDPV2 outbreaks, on advice of the monovalent OPV type 2 (mOPV2) Global Advisory Group, the WHO Director-General releases mOPV2 for outbreak response immunization. Of the administered doses, more than half (51\%) were tOPV and approximately half (47\%) were bOPV; an additional $1.4 \%$ were mOPV2, $0.05 \%$ were IPV plus bOPV, $0.2 \%$ were IPV alone, and $0.15 \%$ were fractional IPV $(0.1 \mathrm{~mL}$ administered intradermally).

In 2017, 172 SIAs were conducted in five WHO regions, during which approximately 1.79 billion total OPV and IPV doses were administered, including 1,110,923,756 (62\%) doses administered during national immunization days, 672,091,158 
Morbidity and Mortality Weekly Report

TABLE 1. Number of supplementary immunization activities (SIAs) conducted, and number of oral poliovirus vaccine (OPV) and inactivated poliovirus (IPV) doses administered, by World Health Organization (WHO) region — worldwide, 2016-2017

\begin{tabular}{|c|c|c|c|c|c|c|c|}
\hline \multirow{2}{*}{$\begin{array}{l}\text { Year/SIAs/Vaccine doses } \\
\text { administered }\end{array}$} & \multicolumn{7}{|c|}{ Region } \\
\hline & Global & AFR & AMR & EMR & EUR & SEAR & WPR \\
\hline \multicolumn{8}{|l|}{2016} \\
\hline SIAs (no.) & 186 & 97 & 0 & 67 & 2 & 14 & 6 \\
\hline \multicolumn{8}{|c|}{ Vaccine (no. of doses administered) } \\
\hline mOPV2 & $28,357,599$ & $28,357,599$ & 0 & 0 & 0 & 0 & 0 \\
\hline bOPV & $940,622,006$ & $274,197,570$ & & $397,909,506$ & $54,880,271$ & $206,507,773$ & $7,126,886$ \\
\hline tOPV & $1,017,074,205$ & $407,366,635$ & 0 & $103,470,392$ & $1,097,605$ & $496,401,815$ & $8,737,758$ \\
\hline IPV & $3,293,021$ & $1,943,763$ & & 134,9258 & 0 & 0 & 0 \\
\hline$I P V+b O P V$ & 904,050 & 0 & 0 & 904,050 & 0 & 0 & 0 \\
\hline fIPV & $2,899,566$ & 0 & 0 & 252,354 & 0 & $2,647,212$ & 0 \\
\hline Total doses & $1,993,150,447$ & $711,865,567$ & 0 & $503,885,560$ & $55,977,876$ & $705,556,800$ & $15,864,644$ \\
\hline \multicolumn{8}{|l|}{2017} \\
\hline SIAs (no.) & 172 & 82 & 0 & 79 & 2 & 8 & 1 \\
\hline \multicolumn{8}{|c|}{ Vaccine (no. of doses administered) } \\
\hline mOPV2 & $70,356,186$ & $65,067,196$ & 0 & $5,288,990$ & 0 & 0 & 0 \\
\hline bOPV & $1,705,913,274$ & $519,920,180$ & 0 & $488,368,342$ & 389,314 & $696,180,796$ & $1,054,642$ \\
\hline tOPV & 0 & 0 & 0 & 0 & 0 & 0 & 0 \\
\hline IPV & $3,522,237$ & 558,897 & 0 & $2,963,340$ & 0 & 0 & 0 \\
\hline IPV + bOPV & $8,920,134$ & & 0 & $8,920,134$ & 0 & 0 & 0 \\
\hline fIPV & 0 & 0 & 0 & 0 & 0 & 0 & 0 \\
\hline Total doses & $1,788,711,831$ & $585,546,273$ & 0 & $505,540,806$ & 389,314 & $696,180,796$ & $1,054,642$ \\
\hline
\end{tabular}

Abbreviations: AFR = African Region, AMR = Region of the Americas; bOPV2 = bivalent oral poliovirus, types 1 and 3; EMR = Eastern Mediterranean Region; $E U R=$ European Region; fIPV = fractional dose inactivated poliovirus vaccine (one fifth of a $0.5 \mathrm{~mL}$ intramuscular dose, given intradermally); IPV $=$ inactivated poliovirus vaccine; $\mathrm{mOPV} 2=$ monovalent oral poliovirus, type 2; SEAR = South-East Asia Region; tOPV2 = trivalent oral poliovirus, types 1, 2, 3; WPR = Western Pacific Region.

(38\%) during subnational immunization days, and 5,696,917 $(0.3 \%)$ during mop-up activities. Of the administered doses, 95\% were bOPV, 3.9\% were mOPV2, 0.5\% were IPV plus bOPV, and $0.2 \%$ were IPV alone.

\section{Poliovirus Surveillance}

Surveillance for acute flaccid paralysis (AFP) is the means of detecting polio cases caused by WPV or cVDPV, confirmed by stool specimen testing through the Global Polio Laboratory Network. The performance of AFP surveillance is assessed through two main indicators: sensitivity and completeness of case investigation. An annual nonpolio AFP rate of $\geq 1$ case per 100,000 population aged $<15$ years for countries in the WHO regions certified as poliofree, or $\geq 2$ for all other countries is considered sufficiently sensitive to detect a case of polio, should it occur. Case investigation is considered to be sufficiently complete if at least $80 \%$ of reported AFP cases have adequate stool specimens collected (i.e., two stool specimens collected $\geq 24$ hours apart, within 14 days of paralysis onset, with arrival at a WHO-accredited laboratory in good condition). In 2016, among the four countries reporting polio cases, three (Afghanistan, Nigeria, Pakistan) met both performance indicators and one (Laos) did not. Among the five countries reporting polio cases in 2017, four (Afghanistan, DRC, Nigeria, Pakistan) met both performance indicators and one (Syria) did not. Although Nigeria and DRC meet AFP surveillance indicators nationally and subnationally in most provinces, both countries are affected by substantial issues in population accessibility and other impediments to AFP surveillance (1). AFP surveillance has been supplemented by environmental surveillance through testing of sewage in many countries, including poliofree countries as well as those with endemic transmission (1).

\section{Reported Poliovirus Cases}

Countries reporting WPV cases. In 2016, 37 WPV cases were detected (Figure): 13 (35\%) in Afghanistan, 20 (54\%) in Pakistan, and four (11\%) in Nigeria. In 2017, 22 WPV cases were identified: 14 (64\%) in Afghanistan and eight (36\%) in Pakistan. No WPV cases have been identified in countries outside of Afghanistan, Nigeria, and Pakistan since 2014. During January 1-March 30, 2018, as of April 24, the low poliovirus transmission season, eight WPV1 cases were reported (seven in Afghanistan; one in Pakistan) (Figure) (Table 2).

Afghanistan reported 13 WPV1 cases in four districts in 2016, compared with 14 WPV1 cases in nine districts in 2017 (7.7\% increase). In 2016, 54\% of WPV1 cases in Afghanistan were reported from Paktika province in the southeastern region. In 2017, 50\% of WPV1 cases were reported from Kandahar province in the southern region. During January 1-March 30, 2018, seven WPV1 cases were detected (four in Kandahar province, one in Nangahar province, and two in Kunar province; the 


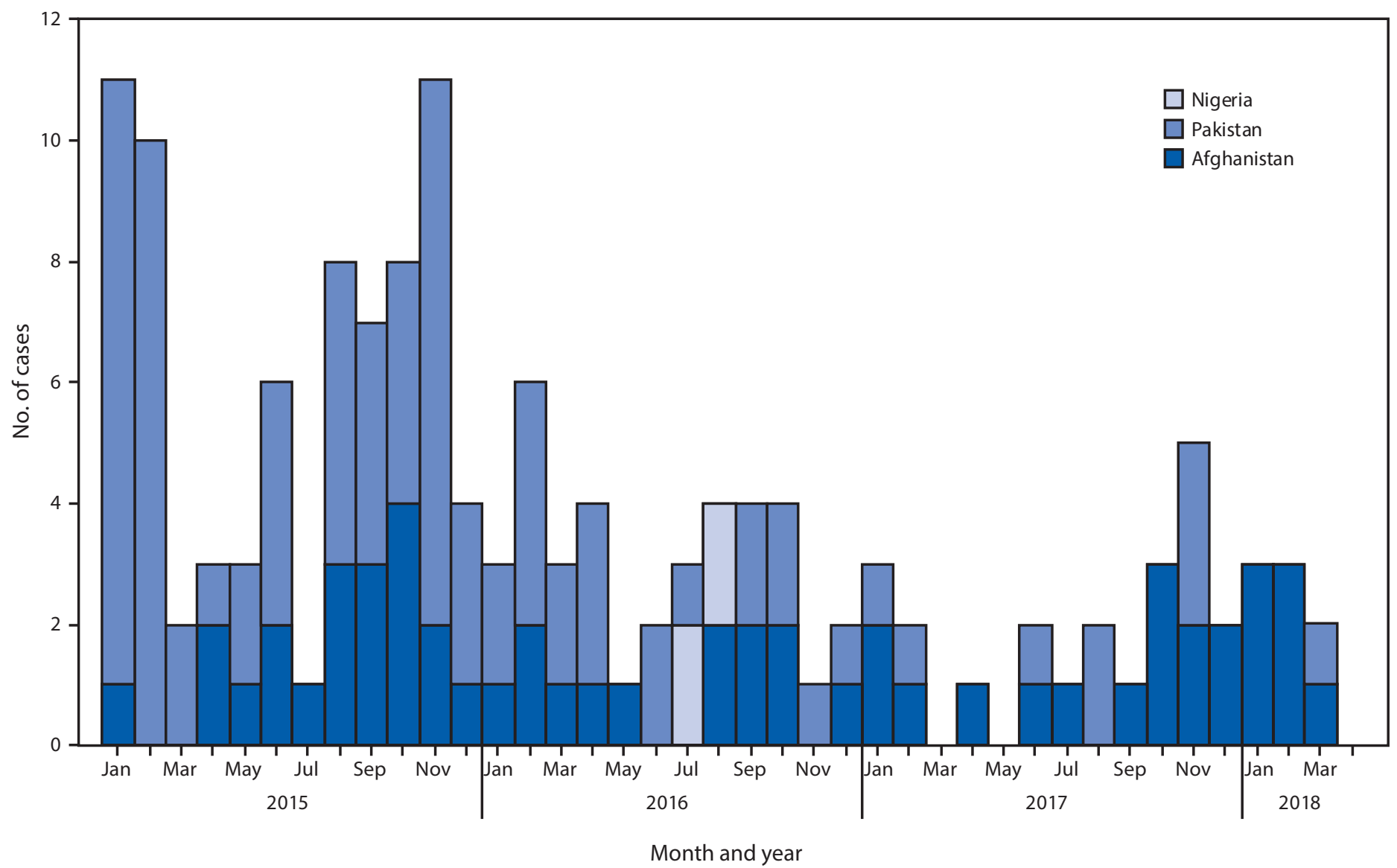

* Data as of April 24, 2018.

latter two provinces are in the eastern region), compared with three WPV1 cases detected during the same period in 2017.

Pakistan reported a $60 \%$ decrease in the number of WPV1 cases, from 20 cases in four districts in 2016 to eight cases in seven districts in 2017. During January 1-March 30, 2018, one WPV1 case was reported (in Balochistan province), compared with two reported during the same period in 2017. WPV1 continues to be isolated from environmental surveillance sites in five provinces of the country (Balochistan, Islamabad, Khyber Pakhtunkhwa, Punjab, and Sindh).

Nigeria reported four WPV1 cases in 2016. No WPV1 cases were reported in 2017 and none to date in 2018.

Countries reporting cVDPV cases and isolations. In 2016, five cVDPV cases were reported from three countries (8). In Laos, an outbreak that began with eight cVDPV type 1 cases in 2015 continued into 2016 with three additional cases reported. One cVDVPV2 case was reported in 2016 in Nigeria and another in Pakistan. In 2017, a total of 96 cVDPV2 cases were reported, including 74 cases from Syria (most recent case in September 2017) and 22 from DRC. The outbreak in
DRC has continued into 2018, with four cases to date, as of April 24, 2018 (the most recent case occurring in February) (9). Isolation of cVDVP2 from environmental samples in Mogadishu, Somalia, in late 2017 and early 2018, and related cVDPV2 from environmental samples in Nairobi, Kenya, in early 2018, has confirmed long-term cVDPV2 transmission, in a broad area, although no associated polio cases have been detected to date. cVDPV type 3 has been isolated in Mogadishu from sewage samples collected in March 2018, again, with no associated polio cases having been detected to date. In Nigeria, cVDPV2 has been recently detected by environmental surveillance in two states in early 2018; no associated polio cases having been detected to date. Response immunization is underway or planned for all these cVDPV cases and isolations.

\section{Discussion}

Although substantial progress was made toward polio eradication during 2016-2017, challenges remain in the countries with endemic transmission. Continued circulation of WPV1 has been confirmed in Afghanistan and Pakistan in the 2018 
TABLE 2. Number of reported polio cases, by country - Worldwide, January 1, 2016-March 30, 2018*

\begin{tabular}{|c|c|c|c|c|c|c|c|c|}
\hline \multirow[b]{2}{*}{ Classification/Country } & \multicolumn{2}{|c|}{2016 (Jan 1-Dec 31) } & \multicolumn{2}{|c|}{2017 (Jan 1-Dec 31) } & \multicolumn{2}{|c|}{2017 (Jan 1-Mar 30) } & \multicolumn{2}{|c|}{2018 (Jan 1-Mar 30) } \\
\hline & WPV & cVDPV & WPV & cVDPV & WPV & cVDPV & WPV & cVDPV \\
\hline \multicolumn{9}{|l|}{ Countries with endemic polio } \\
\hline Afghanistan & 13 & 0 & 14 & 0 & 3 & 0 & 7 & 0 \\
\hline Pakistan & 20 & 1 & 8 & 0 & 2 & 0 & 1 & 0 \\
\hline Nigeria & 4 & 1 & 0 & 0 & 0 & 0 & 0 & 0 \\
\hline Total cases in endemic countries & 37 & 2 & 22 & 0 & 5 & 0 & 8 & 0 \\
\hline \multicolumn{9}{|c|}{ Other countries with reported cVDPV cases } \\
\hline Laos & 0 & 3 & 0 & 0 & 0 & 0 & 0 & 0 \\
\hline Democratic Republic of the Congo & 0 & 0 & 0 & 22 & 0 & 0 & 0 & 3 \\
\hline Syria & 0 & 0 & 0 & 74 & 0 & 0 & 0 & 0 \\
\hline Total cases in other countries & 0 & 3 & 0 & 96 & 0 & 0 & 0 & 3 \\
\hline Total paralytic polio cases & 37 & 5 & 22 & 96 & 5 & 0 & 8 & 3 \\
\hline
\end{tabular}

Abbreviations: $\mathrm{CVDPV}=$ circulating vaccine-derived poliovirus; $\mathrm{WPV}=$ wild poliovirus.

* Data as of April 24, 2018.

low WPV season, and it remains uncertain if WPV circulation has been interrupted in Nigeria (3).

The number of WPV cases in Afghanistan declined from 2015 to 2016, but the decrease did not continue in 2017 . Although negotiations to obtain local access are constantly being undertaken, the number of children who were inaccessible to vaccination in the south and east because of insecurity increased during 2017 (5). In Pakistan, a decline in WPV1 cases since 2014 continued during 2016 and 2017. The detection of WPV in environmental surveillance samples in the absence of WPV-positive AFP cases in several provinces might indicate either surveillance gaps or waning in the intensity of transmission. Intensified SIA schedules and efforts to reach previously unvaccinated children, along with expansion of communitybased initiatives employing local permanent vaccinators and ensuring worker safety have helped reduce the number of WPV cases. Large-scale movement of high-risk populations across Pakistan's border with Afghanistan in both directions continues to pose a challenge to interrupting WPV transmission, and crossborder collaborative vaccination efforts made in 2017 are being enhanced in 2018 (4).

In Nigeria, WPV1 circulation went undetected from mid2014 to mid-2016, and the discovery of both endemic WPV1 and long-standing cVDVP2 transmission in 2016 in Borno State illuminated gaps in surveillance. Continued inaccessibility of insurgent-held areas hinders both immunization and surveillance efforts (3). Enhancement of initiatives for collaborating with the military to reach currently unvaccinated children will be helpful in ensuring interruption of WPV transmission. In the other countries of the Lake Chad basin bordering Borno State (Cameroon, Chad, and Niger), problems with inaccessibility related to insecurity and a large number of difficult-to-access islands have been addressed through progressive improvements in microplanning and implementation

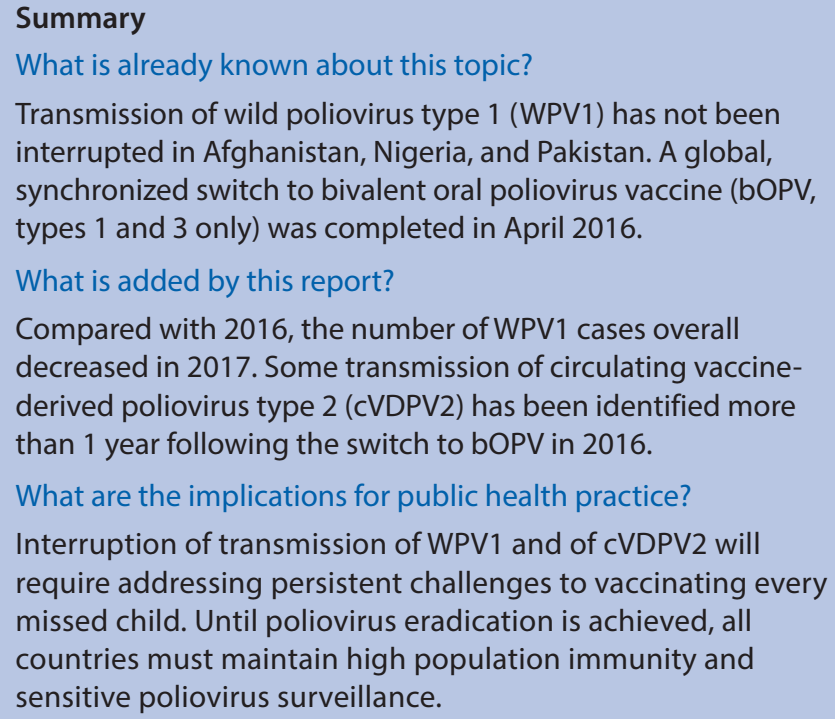

of SIAs, but uncertainties remain regarding SIA quality and success in interrupting undetected WPV transmission.

Global WPV2 eradication was certified in 2015 after no detection since 1999 (2). WPV type 3 has not been detected since 2012 (2). A minimum of 3 years of sensitive AFP surveillance without detection of WPV is required to certify a WHO region as being poliofree (10). Four of six WHO regions (the Region of the Americas, European, South-East Asia, and Western Pacific regions) have been certified free of indigenous WPV. Improvements in AFP surveillance performance in critical subnational areas are required to achieve poliofree certification of the African and Eastern Mediterranean regions.

Because efforts to increase immunity to poliovirus type 2 before the global tOPV to bOPV switch did not reach all persistently unvaccinated children in hard-to-reach areas, some cVDPV2 emergences have been detected following the switch. 
Reaching all children for vaccination in areas with cVDPV2 transmission is also an ongoing challenge.

Although progress toward global polio eradication has continued, challenges in identifying and vaccinating every missed child remain. Much of the recent progress reaching previously missed children has been associated with recruitment of trusted community volunteers who are invested in their locality for vaccination and surveillance efforts. Intensification of efforts to improve the quality of immunization and surveillance activities and to develop additional innovations in addressing persisting challenges is necessary. Until poliovirus eradication is achieved, all countries must remain vigilant by maintaining high population immunity and sensitive poliovirus surveillance.

\section{Acknowledgments}

The Ministries of Health of all countries; World Health Organization (WHO) Regional Office for the Eastern Mediterranean Region, Amman, Jordan; WHO Regional Office for Africa, Brazzaville, Congo; WHO Regional Office for Europe, Copenhagen, Denmark; WHO Regional Office for the Western Pacific, Manila, Philippines; WHO Regional Office for South-East Asia, New Delhi, India; Global Polio Laboratory Network, Geneva, Switzerland; Division of Viral Diseases, National Center for Immunization and Respiratory Diseases, CDC.

\section{Conflict of Interest}

No conflicts of interest were reported.

\footnotetext{
${ }^{1}$ Global Immunization Division, Center for Global Health, CDC; ${ }^{2}$ Polio Eradication Department, World Health Organization, Geneva, Switzerland; ${ }^{3}$ Division of Viral Diseases, National Center for Immunization and Respiratory Diseases, CDC.
}

Corresponding author: Farrah Khan, fkhan@cdc.gov, 425-463-9473.

\section{References}

1. Gardner TJ, Diop OM, Jorba J, Chavan S, Ahmed J, Anand A. Surveillance systems to track progress toward polio eradicationworldwide, 2016-2017. MMWR Morb Mortal Wkly Rep 2018;67:418-23. https://doi.org/10.15585/mmwr.mm6714a3

2. Morales M, Tangermann RH, Wassilak SG. Progress toward polio eradication-worldwide, 2015-2016. MMWR Morb Mortal Wkly Rep 2016;65:470-3. https://doi.org/10.15585/mmwr.mm6518a4

3. Bolu O, Nnadi C, Damisa E, et al. Progress toward poliomyelitis eradication-Nigeria, January-December 2017. MMWR Morb Mortal Wkly Rep 2018;67:253-6. https://doi.org/10.15585/mmwr.mm6708a5

4. Elhamidi Y, Mahamud A, Safdar M, et al. Progress toward poliomyelitis eradication-Pakistan, January 2016-September 2017. MMWR Morb Mortal Wkly Rep 2017;66:1276-80. https://doi.org/10.15585/mmwr. mm6646a4

5. Martinez M, Shukla H, Nikulin J, et al. Progress toward poliomyelitis eradication-Afghanistan, January 2016-June 2017. MMWR Morb Mortal Wkly Rep 2017;66:854-8. https://doi.org/10.15585/mmwr. mm6632a5

6. World Health Organization. WHO vaccine-preventable diseases: monitoring system 2017 global summary. Geneva, Switzerland: World Health Organization; 2017. http://apps.who.int/immunization_ monitoring/globalsummary/timeseries/tswucoveragedtp3.html

7. World Health Organization. Cessation of use of trivalent oral polio vaccine and introduction of inactivated poliovirus vaccine worldwide, 2016. Wkly Epidemiol Rec 2016;91:421-7.

8. Jorba J, Diop OM, Iber J, et al. Update on vaccine-derived poliovirusesworldwide, January 2016-June 2017. MMWR Morb Mortal Wkly Rep 2017;66:1185-91. https://doi.org/10.15585/mmwr.mm6643a6

9. Alleman MM, Chitale R, Burns CC, et al. Vaccine-derived poliovirus outbreaks and events - three provinces, Democratic Republic of the Congo, 2017. MMWR Morb Mortal Wkly Rep 2018;67:300-5. https:// doi.org/10.15585/mmwr.mm6710a4

10. Dowdle WR, Birmingham ME. The biologic principles of poliovirus eradication. J Infect Dis 1997;175(Suppl 1):S286-92. https://doi. org/10.1093/infdis/175.Supplement_1.S286 